\title{
Measurement of the Phase and Intensity Profile of Surface Plasmon Laser Emission
}

\author{
Vasco T. Tenner,* Michiel J. A. de Dood, and Martin P. van Exter \\ Huygens-Kamerlingh Onnes Laboratorium, Leiden University, Niels Bohrweg 2, 2333 CA Leiden, The Netherlands \\ Supporting Information
}

ABSTRACT: We study the near- and far-field radiation patterns of surface plasmon (SP) lasers in metal hole arrays and observe radially polarized vortex-vector laser beams in both near and far field. Besides the intensity profile, also the complementary phase profile is obtained with a beam block experiment, where we block part of the beam in the near field, measure the resulting changes in the far field, and retrieve the phase using an iterative algorithm. This phase profile provides valuable information on the feedback mechanisms and coherence of the laser and shows that our SP laser operates in a phaseslip mode instead of a pure dark mode. To explain our observations, we extend the standard model for distributed feedback lasers by introducing a position dependence in the optical gain and refractive index.

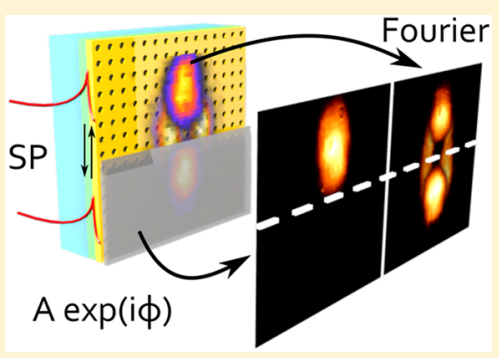

KEYWORDS: surface plasmon laser, distributed feedback, phase retrieval, metal hole array, plasmonic crystal laser, active mirror

ptically coherent laser radiation can be generated if both gain and optical feedback are present in a medium. Our physical understanding of these phenomena originates from comparisons between measured intensity distributions and models of both the amplitude and the phase of the radiation. The optical phase is typically discarded because it evolves too fast to resolve directly with an optical detector or a camera. The inability to measure both amplitude and phase of the emitted laser radiation presents a recurring challenge in optics and limits progress in the field.

More ingenious schemes are needed to observe the phase using slow detectors. One of the simplest schemes uses the mixing of the amplitude and phase information on the light field upon propagation. At the laser exit the amplitude contains information where the light is emitted, while the phase profile contains information about the propagation direction. Recording the intensity distribution on different positions allows retrieval of the phase information by an iterative algorithm. ${ }^{1-3}$

The ability to resolve both amplitude and phase is particularly relevant for lasers that emit nonstandard beam profiles that are not yet fully understood. Examples of such lasers are surface-emitting distributed feedback lasers, such as photonic and plasmonic crystal lasers. Two-dimensional surface-emitting photonic-crystal lasers often emit donut beams with azimuthal polarization, ${ }^{4}$ while surface plasmon lasers create radially polarized vector-vortex beams. ${ }^{5}$ Devices can be tailored to emit other beam shapes, ${ }^{6}$ but information about the phase and amplitude profile is scarce and either has low resolution ${ }^{7}$ or an electrical contact blocks the view. ${ }^{8}$

A better understanding of gain and feedback in plasmonic systems is important for improving photonics applications that use the strong confinement and light-matter interaction provided by plasmons. These applications include ultrasensitive molecule sensors (SERS), 9 anticounterfeiting measures, ${ }^{10}$ perfect absorbers, ${ }^{11}$ ultrafast optical modulators, ${ }^{12}$ and future metal-dielectric metamaterials consisting of arrays of plasmonic subwavelength elements. ${ }^{13,14}$ The strong plasmonic response of passive media is accompanied by ohmic loss due to scattering of the free electrons in the material. Adding media with active gain can resolve this issue, ${ }^{15-17}$ and overcompensation typically leads to laser action, as has been demonstrated in two-dimensional metal particle arrays ${ }^{18}$ and metal hole arrays. ${ }^{5}$

In this Letter, we present the first experimental observation of the phase and amplitude profile of a two-dimensional surface plasmon laser retrieved via the combination of a beam-block experiment and an iterative algorithm. The metal hole array in our study acts as a second-order Bragg grating, which provides a natural output channel and enables easy observation of the intracavity field. Our observations go beyond the standard description of distributed feedback lasers. We extend the standard approach by including a position-dependent gain and refractive index, which are both induced by the optical pump beam, and obtain good agreement between experiment and theory.

\section{DEVICE}

The semiconductor-gold samples that we study contain metal hole arrays with a square lattice, with hole diameters of $160 \mathrm{~nm}$ and a lattice spacing of $470 \mathrm{~nm}$ (see Figure $2 \mathrm{a}$ for a SEM image). The device dimensions studied here are $50 \times 50$ and $100 \times 100 \mu \mathrm{m}$. The Au film is $100 \mathrm{~nm}$ thick and is deposited on a $127 \mathrm{~nm} \mathrm{In}_{x} \mathrm{Ga}_{1-x} \mathrm{As}(x=0.536)$ gain layer on an InP substrate (see Figure 2e for a schematic side cut). Between the gold and the InGaAs, a thin InP spacer layer and a SiN passivation layer

Received: April 6, 2016

Published: May 16, 2016 


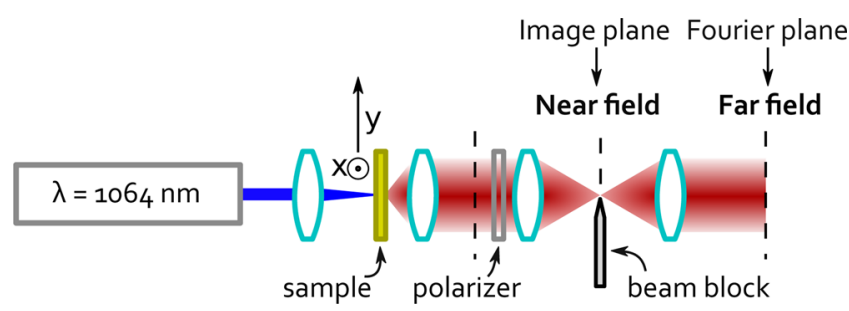

Figure 1. Schematic of the experimental setup to measure the amplitude and phase profile of the laser emission.

were incorporated. The gain layer is sufficiently thin such that the only supported optical mode is the surface plasmon (SP) mode. Similar samples are described in more detail in ref 5 .

\section{EXPERIMENT}

Our experimental geometry is as shown in Figure 1. The sample is mounted in a cryostat with optical access on both sides and cooled to $80 \mathrm{~K}$. We pump the active layer of the sample using a continuous wave laser with a wavelength of $1064 \mathrm{~nm}$ through the transparent InP substrate. The pump beam has a Gaussian profile that can be varied in size between 20 and $50 \mu \mathrm{m}$ full width at half-maximum. The light emitted by the SP laser is collected in transmission on the metal side of the device with a $20 \times$ microscope objective $(\mathrm{NA}=0.4)$ combined with a tube lens $(f=200 \mathrm{~mm})$ to create a $4-f$ imaging system. Hence, the optical field in the image plane is a scaled version of the radiative field at the sample; in this paper, we call this the near field.

In some of the experiments we position a razor blade in the near field to block part of the beam. To inspect the near field, we image it with a lens $(f=100 \mathrm{~mm})$ on a CCD camera.
Subsequently, this lens is replaced by a lens with a longer focal distance $(f=200 \mathrm{~mm})$ such that the far field is retrieved in the back-focal plane. A bandpass filter $(\lambda=1490 \pm 6 \mathrm{~nm})$ that transmits the laser light is used to reduce the broadband spontaneous emission in the measurements.

\section{RESULTS}

Figure 2 show the measured near field (top) and far field (bottom) of the SP laser. Images are shown for the unpolarized light $(b, f)$, with a linear polarizer transmitting $y$-polarized light $(c, g)$, and with half of the near field blocked (d, h). The near field is donut shaped; that is, it is circular with a dark center. The laser area is comparable to the size of the pump, being 40 $\mu \mathrm{m}$ in this case. The dark central spot is remarkable and raises questions about the apparent lack of energy in the center of the device. Figure $2 \mathrm{c}$ shows a polarization-resolved measurement. Since this image rotates along when rotating the polarized axis, we conclude that the near-field donut is radially polarized. The observation of a clear donut in the near field is only apparent when the pump beam is small enough. In our experiments, we observe that larger pump beams (up to $100 \mu \mathrm{m}$ ) result in a larger laser area and spatial inhomogeneity. Nonetheless, there is always a dark spot somewhere, as expected for a topological defect. Under some experimental conditions the laser hops between several spatial modes with different locations of the dark spot, and hence the central dark spot becomes less visible after averaging.

Figure $2 \mathrm{f}-\mathrm{h}$ show the observed far-field intensity profiles and display that the far field is also a radially polarized donut beam. This similarity is not trivial and warrants further investigation. In order to observe the associated phase profile, we perform an experiment in which we block half of the near field with a razor
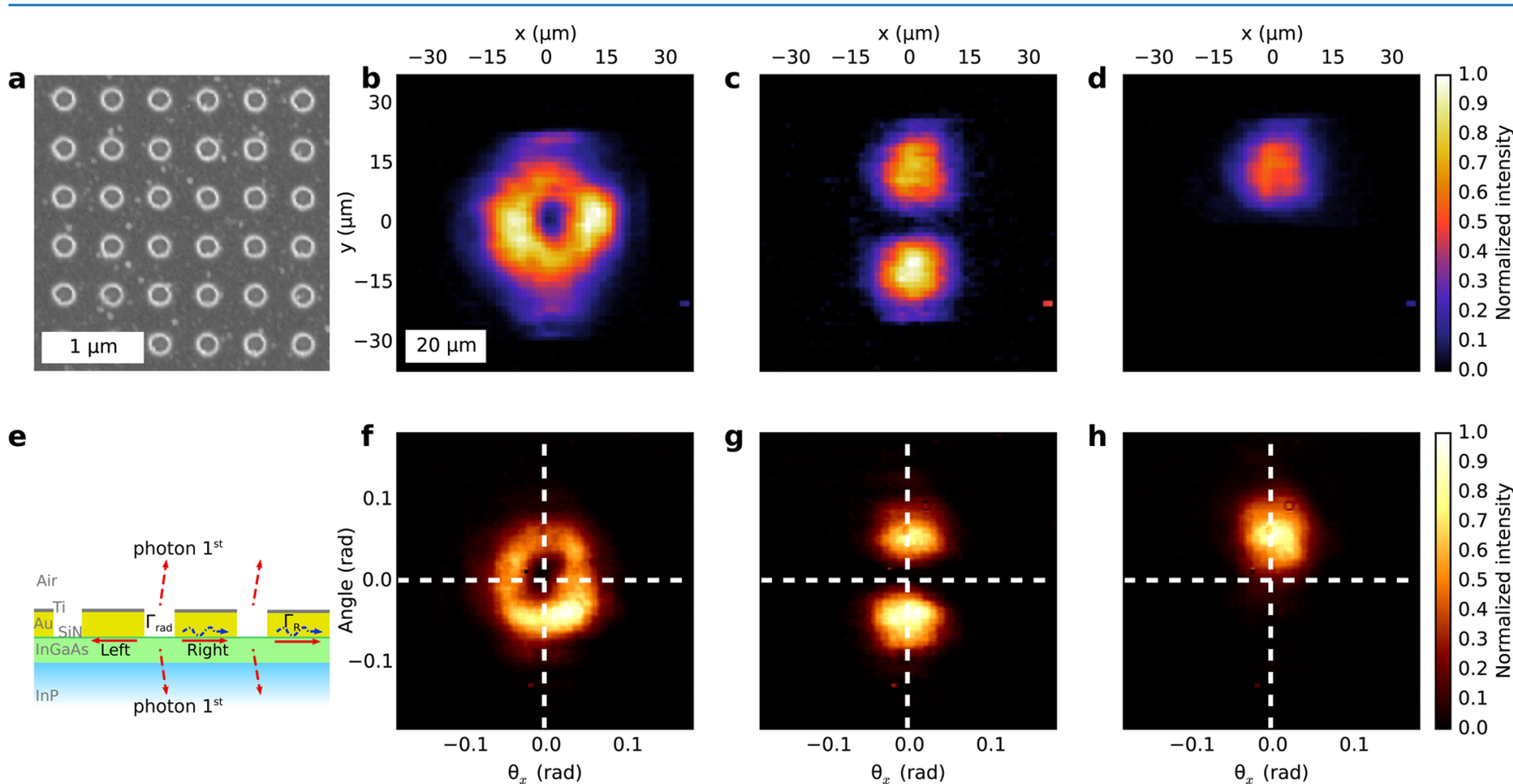

Figure 2. Our SP laser and its emission, measured in near and far field. (a) Scanning electron microscopy image of the metal hole array. (e) Cross section through the sample, indicating the layer stack, the left and right traveling waves, and the emitted light. (b-d) Near-field and (f-h) far-field intensity profiles of a surface plasmon laser. (b, f) Donut-shaped beams in both near and far field. (c, g) Profiles observed behind a polarizer that transmits vertically polarized light. $(\mathrm{d}, \mathrm{h})$ What happens when we block the lower part of the near field. Vertical cross sections are shown in Figure $3 \mathrm{c}, \mathrm{g}$. 

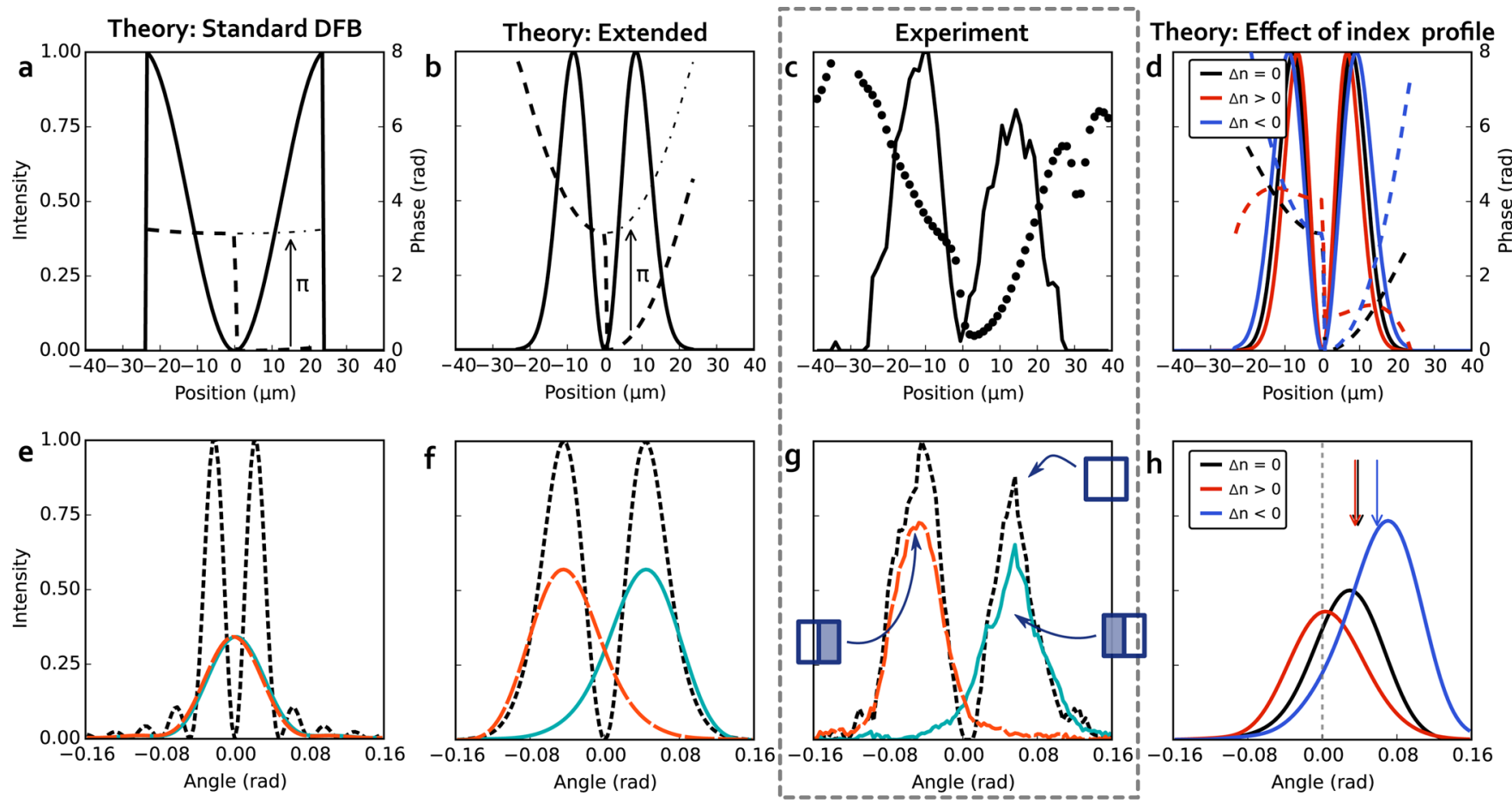

Figure 3. Cross sections through the center along the vertical direction of the $(a-d)$ near field and $(e-h)$ far field. In $(a-d)$, the solid lines show the intensity envelope and the dashed lines show the phase of the near field. To stress the symmetry in (a) and (b), a $\pi$-shifted copy of the phase is displayed as a dashed-dotted line. In $(\mathrm{e}-\mathrm{h})$, the dashed curves show the far-field intensity profiles of the full beam, while the colored curves show the resulting far fields when blocking the right (red dashed) or left (blue solid) half of the near field. The framed figures (c, g) are the experimental data taken from Figure 2 and the reconstructed phase of the near field. The other figures are based on theory: (a, d) Standard DFB theory with uniform gain and index, $(b, f)$ extended theory that includes a position-dependent gain and refractive index, $(\mathrm{d}, \mathrm{h})$ extended theory with variations in the magnitude of the index profile. Note that panel (h) displays the far field for only one blocking condition. The arrows indicate the opening angle of the beam without beam block.

blade and observe the far field. The resulting near field, shown in Figure 2d, is trivial and presented mainly for didactic reasons. The resulting far field, shown in Figure $2 \mathrm{~h}$, depicts that the twolobed far field is now reduced to a single lobe, while the angle of the maximum emission is hardly changed. This observation provides valuable information about the phase profile of the near field, because it indicates the existence of a phase gradient in the near field.

To quantify the full two-dimensional phase profile of the optical field, we retrieve the phase profile with an iterative Gerchberg-Saxton-based algorithm; ${ }^{1}$ see Methods. We find that the retrieved near-field phase exhibits a $\pi$-phase jump in the dark center of the device and exhibits a phase gradient in the radial direction, with a slope that increases toward the edge of the device. A cross section of this phase profile is depicted in Figure $3 c, g$. These figures also show cross sections of the nearand far-field intensity profiles presented earlier in Figure 2. The far field of the full beam has no light in the center, whereas there is emission along the surface normal in the beam block experiments. Because the far field of the full beam should be equal to the coherent sum of the two halves, the dark center in the far field must be formed by interference of emission from the two halves of the sample. This in turn indicates the existence of long-range coherence across the sample. In the rest of this Letter we will discuss the implications of our observations and compare them with theory.

\section{DISCUSSION}

We first compare our results with the standard distributed feedback (DFB) theory ${ }^{19}$ for one-dimensional systems with a finite size. In this theory, the field in the device is decomposed in two traveling waves, which are coupled by scattering at the holes. The relevant parameters are the length $L$ of the device and the coupling rate $\kappa$. The product $\kappa L$ determines the behavior of the laser. This theory yields the threshold condition of the laser: wavelength, gain, and the field profiles of the traveling waves.

The solutions are either symmetric or antisymmetric around the center of the device. In an infinitely large index-coupled system these are dark (nonradiating) and bright (radiating) modes, which are located at the exact center of the Brillouin zone $(k=0){ }^{20-22}$ However, in a "real" laser, the coherence length $l_{\text {coh }}$ is limited by the finite sample size and the scattering, ${ }^{19}$ which breaks the description of a continuous band structure into discrete modes with a detuning from the Bragg wavelength. The relevant modes are at $\Delta k=\pi / l_{\text {coh }}$ and there is no mode at the center of the Brillouin zone.

Scattering in the out-of-plane direction induces radiative loss, which increases the threshold of the radiative solution. ${ }^{23}$ Our device operates in a transverse-magnetic (TM) mode, and hence the coupled mode with the symmetric out-of-plane $E$ field distribution is the nonradiating mode with the lowest threshold, as explained in the Supporting Information.

Figure 3a,b displays the calculated symmetric coupled mode solution of the standard DFB theory for our measured backscatter rate $\kappa / \beta_{0}=0.012,{ }^{21}$ device length $L=50 \mu \mathrm{m}$, 
and refractive index $n_{0}=3.268,{ }^{24}$ corresponding to $\kappa L=8$. The calculated near field shown in Figure 3a contains the essential features of Figure 3c: it has two lobes with opposite sign, indicated by a $\pi$-phase jump in the center of the device. However, in contrast to the experiment the calculated phase in each of the lobes is almost constant. As a consequence, the farfield profiles depicted in Figure $3 \mathrm{e}$ are very different from the observations depicted in Figure 3g: the calculated profile is too narrow and is oscillatory at larger angles. Furthermore, the emission by half of the device is incorrectly predicted to be a single lobe located close to the surface normal.

To explain our observations, we extend the standard DFB theory by introducing a position dependence of the gain and refractive index (see Supporting Information for derivation). Both are mainly set by the carrier density, which is position dependent due to inhomogeneous pumping and diffusion, and to a lesser extent by the local temperature associated with heating of the device. We model the local gain and index as the Gaussian profile of the pump beam and note that deviations from an exact Gaussian shape are unimportant. In the center of the pumped area there is an effective gain, as discussed below, while outside the pumped area there is an effective loss (negative gain), which is mainly caused by absorption in the gain layer. We solve the coupled mode equations by an active mirror approach, ${ }^{25}$ which is relatively simple and powerful, as explained in the Supporting Information. The resulting fields at the threshold are shown in Figure 3b,f. The phase in the near field now increases toward the edge of the samples, very comparable to our measurements. Hence, also the far-field profiles of the beam block experiment are very similar to our observations: the maxima are now at the same angle as the lobes of the full beam.

Figure 3d,h illustrates the predicted effect of index guiding and antiguiding on the laser, with a Gaussian gain and index profile, with $\Delta n=0, \Delta n=+0.13$, and $\Delta n=-0.13$, between the pumped center and the unpumped edges. The differences are best visible in a beam block experiment (see Figure $3 \mathrm{~h}$ ), where the angle of the maxima of the lobe moves inward for $\Delta n>0$ and outward for $\Delta n<0$. The near fields depicted in Figure 3d show index guiding for $\Delta n>0$ and index antiguiding for $\Delta n<$ 0 . This guiding can also be interpreted as a plasmonic band gap with spatial dependence. ${ }^{26,27}$ Our experimental data can be best fitted with $\Delta n=-0.05$, which is consistent with the typical refractive index changes of pumped bulk material. ${ }^{28}$

The gain profile, in contrast to the index profile, leads to guiding, because the effective gain in the center of the pumped area is higher than its surroundings. The gain and loss used in the model have realistic values: The unpumped areas have an effective intensity loss of $243000 \mathrm{~cm}^{-1}$. At the threshold, the net gain in the center is $\sim 340 \mathrm{~cm}^{-1}$. The required material intensity gain is the sum of the net gain and the ohmic loss of our device with transparent $\operatorname{InP}^{24}\left(270 \mathrm{~cm}^{-1}\right)$, divided by the confinement factor in the gain layer ${ }^{15}(0.32)$, and it is around $2000 \mathrm{~cm}^{-1}$, which is a reasonable number for a semiconductor operated at high carrier densities. ${ }^{29,30}$

For completeness we note that we have used a onedimensional model to describe a two- (or even three)dimensional system. Hence the derived numbers may differ somewhat from reality. From literature on DFB theory in two dimensions, ${ }^{31,32}$ we expect that the influence of such $2 \mathrm{D}$ coupling on the derived numbers is rather low in our system, because it already operates in the overcoupled regime and the $2 \mathrm{D}$ coupling is small compared to the $1 \mathrm{D}$ coupling. In earlier work ${ }^{21,24}$ we measured $k_{2} / k_{3} \approx 0.3$, where $k_{2}$ and $k_{3}=\kappa$ are the scattering rates of respectively $90^{\circ}$ and $180^{\circ}$ scattering. ${ }^{31}$ This extra coupling will only marginally change the detuning ${ }^{32}$ and threshold. ${ }^{31}$ Other authors have extended the standard DFB theory to two dimensions to answer the question under which conditions the symmetric mode can lase. ${ }^{33}$ These analyses confirm that 2D DFB lasers are expected to emit donut-shaped beams.

\section{CONCLUSION}

This paper reports the first measurement and reconstruction of the phase and the amplitude of surface plasmon laser emission. Our two-dimensional plasmonic crystal emits donut-shaped and radially polarized light, both in the near field and in the far field. By blocking half of the laser emission we retrieve the phase of the emission and demonstrate the existence of longrange coherence and lasing in a symmetric nonradiative mode. Our observations cannot be explained with the standard DFB theory, which assumes a device with uniform properties. We extend this theory by introducing position dependence of the gain and refractive index and find good agreement with our measurements on surface-emitting DFB lasers. This provides the following three insights: First, due to the round trip phase condition, the laser is in a phase-slip mode and not in a dark mode, and hence the laser can radiate. Second, we attribute the lack of emission in the center of the near field to interference between in-plane counterpropagating waves. Third, the central zero in the far field also results from the symmetry of the lasing mode and demonstrates the existence of long-range coherence over the full sample.

Our results demonstrate a powerful method to analyze surface-emitting lasers. This method can also be deployed on surface plasmon lasers with other lattice symmetries or on photonic-crystal lasers in order to understand and improve their characteristics. Furthermore, our results indicate that much of the current knowledge about one- and twodimensional photonic-crystal lasers can be applied to understand and improve surface plasmon lasers.

\section{METHODS}

The phase of the fields is retrieved with an iterative Gerchberg-Saxton-based algorithm. ${ }^{1-3}$ The near- and farfield measurements are used as support for the algorithm. The phase is retrieved for three sets of conjugate measurements in parallel: (i) the full polarization resolved measurements shown in Figure $2 c, g$ and measurements with either a blocked (ii) bottom (Figure 2d,h) or (iii) top (measurements not shown). Every fifth iteration, the reconstructed phase of alternately the bottom or top part is applied on the full near-field reconstruction. After 30 iterations, we end with 10 iterations on the full fields. The algorithm converges and is stable to noise.

\section{ASSOCIATED CONTENT}

\section{Supporting Information}

The Supporting Information is available free of charge on the ACS Publications website at DOI: 10.1021/acsphotonics.6b00239.

A derivation of the extended DFB theory, with an explanation of how the resulting equations can be solved in the time domain and spatial domain and a comparison with previous efforts (PDF) 


\section{AUTHOR INFORMATION}

\section{Corresponding Author}

*E-mail: tenner@physics.leidenuniv.nl.

\section{Notes}

The authors declare no competing financial interest.

\section{ACKNOWLEDGMENTS}

The authors thank Dirk E. Boonzajer Flaes for his discussions about the iterative algorithm, Matthias Saba for his discussions about surface plasmon lasers, and Peter J. van Veldhoven and Erik Jan Geluk for their help in fabricating the samples at the COBRA Research Institute of the Technische Universiteit Eindhoven, The Netherlands. This work is part of the research program of the Foundation for Fundamental Research on Matter (FOM), which is part of The Netherlands Organization for Scientific Research (NWO).

\section{REFERENCES}

(1) Gerchberg, R. W.; Saxton, W. O. Practical Algorithm for Determination of Phase from Image and Diffraction Plane Pictures. Optik 1972, 35, 237-246.

(2) Fienup, J. R. Phase retrieval algorithms: a personal tour [Invited]. Appl. Opt. 2013, 52, 45-56.

(3) Rodenburg, J. M.; Faulkner, H. M. L. A phase retrieval algorithm for shifting illumination. Appl. Phys. Lett. 2004, 85, 4795-4797.

(4) Sakai, K.; Miyai, E.; Sakaguchi, T.; Ohnishi, D.; Okano, T.; Noda, $\mathrm{S}$. Lasing band-edge identification for a surface-emitting photonic crystal laser. IEEE J. Select. Areas Comm. 2005, 23, 1335-1340.

(5) van Beijnum, F.; van Veldhoven, P. J.; Geluk, E. J.; de Dood, M. J. A.; 't Hooft, G. W.; van Exter, M. P. Surface Plasmon Lasing Observed in Metal Hole Arrays. Phys. Rev. Lett. 2013, 110, 206802.

(6) Miyai, E.; Sakai, K.; Okano, T.; Kunishi, W.; Ohnishi, D.; Noda, S. Photonics: Lasers producing tailored beams. Nature 2006, 441, 946-946.

(7) Notomi, M.; Suzuki, H.; Tamamura, T. Directional lasing oscillation of two-dimensional organic photonic crystal lasers at several photonic band gaps. Appl. Phys. Lett. 2001, 78, 1325.

(8) Imada, M.; Chutinan, A.; Noda, S.; Mochizuki, M. Multidirectionally distributed feedback photonic crystal lasers. Phys. Rev. B 2002, 65, 195306.

(9) Fleischmann, M.; Hendra, P. J.; McQuillan, A. J. Raman spectra of pyridine adsorbed at a silver electrode. Chem. Phys. Lett. 1974, 26, $163-166$.

(10) Cui, Y.; Hegde, R. S.; Phang, I. Y.; Lee, H. K.; Ling, X. Y. Encoding molecular information in plasmonic nanostructures for anticounterfeiting applications. Nanoscale 2014, 6, 282-288.

(11) Liu, N.; Mesch, M.; Weiss, T.; Hentschel, M.; Giessen, H. Infrared Perfect Absorber and Its Application As Plasmonic Sensor. Nano Lett. 2010, 10, 2342-2348.

(12) Haffner, C.; et al. All-plasmonic Mach-Zehnder modulator enabling optical high-speed communication at the microscale. Nat. Photonics 2015, 9, 525-528.

(13) Garcia-Vidal, F. J.; Martín-Moreno, L.; Pendry, J. B. Surfaces with holes in them: new plasmonic metamaterials. J. Opt. A: Pure Appl. Opt. 2005, 7, S97.

(14) Meinzer, N.; Barnes, W. L.; Hooper, I. R. Plasmonic meta-atoms and metasurfaces. Nat. Photonics 2014, 8, 889-898.

(15) van Beijnum, F.; van Veldhoven, P. J.; Jan Geluk, E.; 't Hooft, G. W.; van Exter, M. P. Loss compensation of extraordinary optical transmission. Appl. Phys. Lett. 2014, 104, 061112.

(16) Hess, O.; Pendry, J. B.; Maier, S. A.; Oulton, R. F.; Hamm, J. M.; Tsakmakidis, K. L. Active nanoplasmonic metamaterials. Nat. Mater. 2012, 11, 573-584.

(17) Baumberg, J.; Noginov, M.; Aizpurua, J.; Lin, K.; Ebbesen, T.; Kornyshev, A. A.; Sapienza, R.; van Hulst, N.; Kotni, S.; García de Abajo, F. J.; Ginzburg, P.; Hess, O.; Brongersma, M.; Bozhevolnyi, S.
Quantum plasmonics, gain and spasers: general discussion. Faraday Discuss. 2015, 178, 325-334.

(18) Zhou, W.; Dridi, M.; Suh, J. Y.; Kim, C. H.; Co, D. T.; Wasielewski, M. R; Schatz, G. C.; Odom, T. W. Lasing action in strongly coupled plasmonic nanocavity arrays. Nat. Nanotechnol. 2013, $8,506-511$.

(19) Kogelnik, H.; Shank, C. V. Coupled-Wave Theory of Distributed Feedback Lasers. J. Appl. Phys. 1972, 43, 2327.

(20) Ropers, C.; Park, D.; Stibenz, G.; Steinmeyer, G.; Kim, J.; Kim, D.; Lienau, C. Femtosecond Light Transmission and Subradiant Damping in Plasmonic Crystals. Phys. Rev. Lett. 2005, 94, 113901.

(21) van Exter, M. P.; Tenner, V. T.; van Beijnum, F.; de Dood, M. J. A.; van Veldhoven, P. J.; Geluk, E. J.; 't Hooft, G. W. Surface plasmon dispersion in metal hole array lasers. Opt. Express 2013, 21, 27422.

(22) Joannopoulos, J. D.; Johnson, S. G.; Winn, J. N.; Meade, R. D. Photonic Crystals: Molding the Flow of Light (Second ed.); Princeton University Press, 2011.

(23) Streifer, W.; Burnham, R. D.; Scifres, D. R. Radiation losses in distributed feedback lasers and longitudinal mode selection. IEEE J. Quantum Electron. 1976, 12, 737-739.

(24) Tenner, V. T.; Delft, A. N. v.; Dood, M. J. A. d.; Exter, M. P. v. Loss and scattering of surface plasmon polaritons on optically-pumped hole arrays. J. Opt. 2014, 16, 114019.

(25) Tromborg, B.; Lassen, H.; Olesen, H. Traveling wave analysis of semiconductor lasers: modulation responses, mode stability and quantum mechanical treatment of noise spectra. IEEE J. Quantum Electron. 1994, 30, 939-956.

(26) Chassagneux, Y.; Colombelli, R.; Maineult, W.; Barbieri, S.; Khanna, S. P.; Linfield, E. H.; Davies, A. G. Graded photonic crystal terahertz quantum cascade lasers. Appl. Phys. Lett. 2010, 96, 031104.

(27) Xu, G.; Colombelli, R.; Khanna, S. P.; Belarouci, A.; Letartre, X.; Li, L.; Linfield, E. H.; Davies, A. G.; Beere, H. E.; Ritchie, D. A. Efficient power extraction in surface-emitting semiconductor lasers using graded photonic heterostructures. Nat. Commun. 2012, 3, 952.

(28) Morthier, G.; Vankwikelberge, P. Handbook of Distributed Feedback Laser Diodes, Second ed.; Artech House, 2013.

(29) Hill, M. T. Status and prospects for metallic and plasmonic nano-lasers [Invited]. J. Opt. Soc. Am. B 2010, 27, B36-B44.

(30) Berini, P.; De Leon, I. Surface plasmon-polariton amplifiers and lasers. Nat. Photonics 2011, 6, 16-24.

(31) Sakai, K.; Miyai, E.; Noda, S. Two-dimensional coupled wave theory for square-lattice photonic-crystal lasers with TM-polarization. Opt. Express 2007, 15, 3981-3990.

(32) Sakai, K.; Miyai, E.; Noda, S. Coupled-Wave Theory for SquareLattice Photonic Crystal Lasers With TE Polarization. IEEE J. Quantum Electron. 2010, 46, 788-795.

(33) Vurgaftman, I.; Meyer, J. Design optimization for highbrightness surface-emitting photonic-crystal distributed-feedback lasers. IEEE J. Quantum Electron. 2003, 39, 689-700. 